\title{
Random attractors for non-autonomous stochastic wave equations with nonlinear damping and white noise
}

\author{
Huazhen Yao' and Jianwen Zhang ${ }^{2 *}$
}

\section{"Correspondence:}

1489088560@qq.com

${ }^{2}$ College of Mathematics, Taiyuan

University of Technology, Taiyuan

P.R. China

Full list of author information is

available at the end of the article

\begin{abstract}
This paper is concerned with the asymptotic behavior of solutions to a non-autonomous stochastic wave equation with additive white noise, for which the nonlinear damping has a critical cubic growth rate. By showing the pullback asymptotic compactness of the stochastic dynamic systems, we prove the existence of a random attractor in $H_{0}^{1} \times L^{2}$.
\end{abstract}

Keywords: Random attractors; Additive white noise; Nonlinear damping; Non-autonomous stochastic wave equation

\section{Introduction}

This paper deals with the existence of random attractors for the following nonautonomous stochastic wave equation with white noise in a bounded domain $U \subset R^{3}$ with smooth boundary $\partial U$ :

$$
\begin{aligned}
& u_{t t}+q(u) u_{t}+\alpha u_{t}-\Delta u+f(u, x)=g(x, t)+a h(x) \dot{W}(t), \\
& \left.u(x, t)\right|_{x \in \partial U}=0, \quad t \geq \tau, \tau \in R, \\
& u(x, \tau)=u_{0 \tau}(x), \quad u_{t}(x, \tau)=u_{1 \tau}(x), \quad x \in U, \tau \in R,
\end{aligned}
$$

for $(x, t) \in U \times(\tau,+\infty)$ with $\tau \in R$, where $h \in H_{0}^{1}(U) \cap H^{2}(U)$ and $\alpha \geq 0$ is the damping coefficient. Here $u(x, t)$ is a real-valued function on $U \times[\tau,+\infty) ; g(x, \cdot) \in C_{b}\left(R, H_{0}^{1}(U)\right)$ is a time-dependent driving force; $C_{b}\left(R, H_{0}^{1}(U)\right)$ denotes the set of continuous bounded functions from $R$ into $H_{0}^{1}(U)$; and $W(t)$ is a two-sided real-valued Wiener processes on the probability space $(\Omega, \mathcal{F}, P)$. In addition, the function $q: R \rightarrow R$ and the nonlinear function $f$ satisfy the following assumptions:

$\left(H_{1}\right)$ The function $q \in \mathcal{C}^{1}$ is not identically equal to zero, and there exist three constants $\alpha_{1}, \alpha_{2}, \alpha_{3}$ and $\alpha_{2} \geq\left|\alpha_{1}\right|$ such that

$$
-\alpha<\alpha_{1} \leq q(s) \leq \alpha_{2}<+\infty, \quad\left|q^{\prime}(s)\right| \leq \alpha_{3}, \quad \forall s \in R .
$$

(c) The Author(s) 2020. This article is licensed under a Creative Commons Attribution 4.0 International License, which permits use, sharing, adaptation, distribution and reproduction in any medium or format, as long as you give appropriate credit to the original author(s) and the source, provide a link to the Creative Commons licence, and indicate if changes were made. The images or other third party material in this article are included in the article's Creative Commons licence, unless indicated otherwise in a credit line to the material. If material is not included in the article's Creative Commons licence and your intended use is not permitted by statutory regulation or exceeds the permitted use, you will need to obtain permission directly from the copyright holder. To view a copy of this licence, visit http://creativecommons.org/licenses/by/4.0/. 
$\left(H_{2}\right)$ Let $f(u, x)=f_{1}(u, x)+f_{2}(u, x)$ and $F_{i}=\int_{0}^{u} f_{i}(r, x) d r$, where $f_{1}(\cdot, x) \in \mathcal{C}^{2}(R, R), f_{2}(\cdot, x) \in$ $\mathcal{C}^{1}(R, R)$. Furthermore, $f_{1}, f_{2}$ meet conditions that there exist constants $c_{1}, c_{2}, c_{3}, c_{4}>$ 0 and functions $\phi_{i}(x) \in L^{1}(U), i=1,2$, such that

$$
\begin{aligned}
& f_{1, u}^{\prime}(0, x)=0, \quad f_{1}(u, x) u \geq 0, \quad\left|f_{1, u}^{\prime \prime}(u, x)\right| \leq c_{1}(1+|u|), \\
& f_{2}(0, x)=0, \quad\left|f_{2, u}^{\prime}(u, x)\right| \leq c_{2}\left(1+|u|^{p}\right), \quad 0 \leq p \leq 2, \\
& c_{3} u^{4}-\phi_{1}(x) \leq F_{i}(u, x) \leq c_{4} u f_{i}(u, x)+\phi_{2}(x), \quad \forall u \in R, x \in U .
\end{aligned}
$$

In the deterministic damped wave equation (i.e., $a=0$ ), global attractors have been studied by many authors, such as [1-3] and the reference therein. In addition, uniform attractors and pullback attractors also attracted many experts' attention, cf. [4-8]. If the function $g$ does not depend on time, (1)-(3) is an autonomous stochastic wave equation, and its random attractors have been explored in [9-13]. For many problems, such as wave propagation through the atmosphere or the ocean, the more realistic models must take the random fluctuation into account. So it is important and interesting to study random attractors. For non-autonomous random dynamical systems, Wang established an efficacious theory about the existence of random attractors [14-17]. Particularly, Li [18] studied the asymptotic dynamics for a stochastic damped wave equation with multiplicative noise defined on unbounded domains and proved the existence of random attractors. For the non-autonomous stochastic strongly damped wave equation, the existence of random attractors is proved in [19-21]. Lv and Wang [10] also studied the existence of random attractors for the stochastic wave equation and showed the upper semicontinuous dependence of the random attractor on parameters. The authors in [22] studied the asymptotic behavior of a class of non-autonomous nonlocal fractional stochastic parabolic equations driven by multiplicative white noise on the entire space $R^{n}$.

In this paper, (1)-(3) is a non-autonomous stochastic system where the external term $g$ is time-dependent. We shall transform the stochastic wave equation into a deterministic one with random parameter and random initial data through an Ornstein-Uhlenbeck process $z\left(\theta_{t} \omega\right)$, then prove the existence of a random attractor for the random dynamical system generated by (1)-(3). It is well known that the key step in proving the existence of attractors in both deterministic and random systems is to establish the compactness of the system in some sense. Motivated by [23], we will work out this problem.

The paper is arranged as follows. In Sect. 2, we collect some basic concepts and background material about random attractor for the random dynamical system generated by (1)-(3), then the existence and uniqueness of solutions is established in Sect. 3. In Sect. 4, we consider the concrete bounds of the solution and decompose the solution of (12)-(13) into two parts. In Sect. 5, we establish the asymptotic compactness of the random dynamical system and obtain the existence of the random attractor.

\section{Random dynamical systems}

In this section, we collect some basic definitions and known results about general random dynamical systems (see [17, 24, 25] for details).

Let $(\Omega, \mathcal{F}, P)$ be a probability space, where $\Omega=\{\omega \in C(R, R): \omega(0)=0\}$ is endowed with compact-open topology. $\mathcal{F}$ is the Borel $\sigma$-algebra on $\Omega$ and $P$ is the corresponding Wiener 
measure on $\mathcal{F}$. For any $t$, let $\left(\theta_{t}\right)_{t \in R}$ on $\Omega$ via

$$
\theta_{t} \omega(\cdot)=\omega(\cdot+t)-\omega(t), \quad t \in R,
$$

thus $\left(\Omega, \mathcal{F}, P,\left(\theta_{t}\right)_{t \in R}\right)$ is an ergodic metric dynamical system [24]. In the following, $X$ labels as a Banach or Hilbert space with the Borel $\sigma$-algebra $B(X)$.

Definition 2.1 Let $\left\{\theta_{t}\right\}_{t \in R}$ be a family of $(B(R \times \mathcal{F}), \mathcal{F})$-measurable mappings, $\theta_{t}: R \times$ $\Omega \rightarrow \Omega$ such that $\theta_{0}(\cdot)$ is the identity on $\Omega, \theta_{s+t}(\cdot)=\theta_{t}(\cdot) \circ \theta_{s}(\cdot)$ for all $t, s \in R$ and $P \theta_{t}=P$ for all $t \in R$.

Definition 2.2 Let $\left(\Omega, \mathcal{F}, P,\left(\theta_{t}\right)_{t \in R}\right)$ be a parametric dynamical system. A mapping $\Phi$ : $R^{+} \times R \times \Omega \times X \rightarrow X$ is called a continuous cocycle on $X$ over $R$ and $\left(\Omega, \mathcal{F}, P,\left(\theta_{t}\right)_{t \in R}\right)$ if, for all $\tau \in R, \omega \in \Omega$, and $t, s \in R^{+}$, the following conditions (i)-(iv) are satisfied:

(i) $\Phi(\cdot, \tau, \cdot, \cdot): R^{+} \times \Omega \times X \rightarrow X$ is a $\left(B\left(R^{+}\right) \times \mathcal{F} \times B(X), B(X)\right)$-measurable mapping;

(ii) $\Phi(0, \tau, \omega, \cdot)$ is the identity on $X$;

(iii) $\Phi(t+s, \tau, \omega, \cdot)=\Phi\left(t, \tau+s, \theta_{s} \omega, \cdot\right) \circ \Phi(s, \tau, \omega, \cdot)$;

(iv) $\Phi(t, \tau, \omega, \cdot): X \rightarrow X$ is continuous.

\section{Definition 2.3}

(1) Let $2^{X}$ be the collection of all subsets of $X$. A set-valued mapping $(\tau, \omega) \rightarrow D(\tau, \omega): R \times \Omega \rightarrow 2^{X}$ is called measurable with respect to $\mathcal{F}$ in $\Omega$ if $D(\tau, \omega)$ is a (usually closed) nonempty subset of $X$ and the mapping $\omega \in \Omega \rightarrow d(x, D(\tau, \omega))$ is $(\mathcal{F}, \mathcal{B}(\mathcal{R}))$-measurable for every fixed $x \in X$ and $\tau \in R$, then $D=D(\tau, \omega): \tau \in R, \omega \in \Omega$ is called a random set.

(2) Let $D$ be a collection of random sets in a Polish space $X$. A continuous cocycle $\Phi$ is said to be pullback $\mathcal{D}$-asymptotically compact ( $\mathcal{D}$-a.c.) in $X$ if, for any $\tau \in R, \omega \in \Omega$, $D \in \mathcal{D}$ and any sequences $t_{n} \rightarrow+\infty, x_{N} \in D\left(\tau-t_{n}, \theta_{-t_{n}} \omega\right)$, the sequence $\Phi\left(t_{n}, \tau-t_{n}, \theta_{-t_{n}} \omega, x_{n}\right)$ has a convergent subsequence in $X$.

(3) Let $K=K(\tau, \omega): \tau \in R, \omega \in \Omega \in D$. Then $K$ is called a pullback $\mathcal{D}$-absorbing set for $\Phi$ if, for all $\tau \in R, \omega \in \Omega$ and for every $D \in \mathcal{D}$, there exists $t_{0}(K, \tau, \omega)>0$ such that $\Phi\left(t, \tau-t, \theta_{-t} \omega, D\left(\tau-t, \theta_{-t} \omega\right)\right) \subset K(\tau, \omega)$ for any $t \geq t_{0}$.

(4) A family $C=C(\tau, \omega): \tau \in R, \omega \in \Omega \in \mathcal{D}$ is said to be pullback $\mathcal{D}$-attracting if $\lim _{t \rightarrow+\infty} d\left(\Phi\left(t, \tau-t, \theta_{-t} \omega, D\left(\tau-t, \theta_{-t} \omega\right), C(\tau, \omega)\right)\right)=0$ for all $D \in \mathcal{D}$.

(5) A compact set $A=\{A(\tau, \omega): \tau \in R, \omega \in \Omega\} \in \mathcal{D}$ is called a pullback $\mathcal{D}$-attractor for $\Phi$ if $A$ attracts every $B \in \mathcal{D}$ and $A$ is invariant in the sense that $\Phi(t, \tau, \omega, A(\tau, \omega))=A\left(\tau+t, \theta_{t} \omega\right)$ for every $t \geq 0, \tau \in R$, and $\omega \in \Omega$.

In addition, if there exists $T>0$ such that $A(\tau+T, \omega)=A(\tau, \omega)$ for any $\tau \in R, \omega \in \Omega$, then $A$ is periodic with period $T$.

Proposition 2.1 Let D be a neighborhood-closed collection of $(\tau, \omega)$-parametrized families of nonempty subsets of $X$ and $\Phi$ be a continuous cocycle on $X$ over $R$ and $\left(\Omega, \mathcal{F}, P,\left\{\theta_{t}\right\}_{t \in R}\right)$. Then $\Phi$ has a pullback D-attractor $A$ in $D$ if and only if $\Phi$ is pullback $\mathcal{D}$-asymptotically compact in $X$ and $\Phi$ has a closed $\mathcal{F}$-measurable pullback $\mathcal{D}$-absorbing set $K$ in $D$. The unique pullback $D$-attractor $A=A(\tau, \omega)$ is given by

$$
A(\tau, \omega)=\bigcap_{r \geq 0} \Phi\left(t, \tau-t, \theta_{-t} \omega\right), K\left(\tau-t, \theta_{-t} \omega\right), \quad \tau \in R, \omega \in \Omega .
$$


Proposition 2.2 Let D be a neighborhood-closed collection of $(\tau, \omega)$-parametrized families of nonempty subsets of $X$. If $\Phi$ is a continuous $\tau$-periodic cocycle with period $T>0$ on $X$ over $R$ and $\left(\Omega, \mathcal{F}, P,\left\{\theta_{t}\right\}_{t \in R}\right)$ and if $\Phi$ has a pullback $\mathcal{D}$-attractor $A \in D$, then $A$ is $\tau$-periodic with period $T$ if and only if $\Phi$ has a closed $\mathcal{F}$-measurable pullback $\mathcal{D}$-absorbing set $K \in D$ with $K=K(\tau, \omega)$ being periodic in $\tau$ with period $T$ for each $\omega \in \Omega$.

Notation Set $E=H_{0}^{1}(U) \times L^{2}(U)$ with its inner product and norm as follows:

$$
\left(z_{1}, z_{2}\right)_{E}=\left(\left(u_{1}, u_{2}\right)\right)+\left(v_{1}, v_{2}\right), \quad\|z\|_{E}=(z, z)_{E}^{\frac{1}{2}}
$$

for all $z_{i}=\left(u_{i}, v_{i}\right)^{T}, i=1,2$, and $z=(u, v)^{T}$ in $E$.

$$
(u, v)=\int_{U} u(x) v(x) d x, \quad\|u\|=\|u\|_{L^{2}}=(u, u)^{\frac{1}{2}}
$$

for all $u, v \in L^{2}(U)$, and

$$
((u, v))=\int_{U} \nabla u(x) \nabla v(x) d x, \quad\|u\|_{1}=\|u\|_{H_{1}^{0}}=((u, u))^{\frac{1}{2}}
$$

for all $u, v \in H_{0}^{1}(U)$. More generally, denote $E_{s}=W^{s, 2}(U) \cap H_{0}^{1} \times W^{s-1,2}(U)$ for $s \in R$.

The letters $c$ and $c_{i}(i=1,2, \ldots)$ are generic positive constants which do not depend on $\omega, \tau, t, a$.

\section{Existence and uniqueness of solutions}

In this section, motivated by $[26,27]$, we establish the existence and uniqueness of solutions for Eqs. (1)-(3). Let $\lambda$ be the first eigenvalue of the operator $A:=-\Delta$ on $U$ with Dirichlet boundary conditions. Note that $A: H_{0}^{1}(U) \cap H^{2}(U) \rightarrow L^{2}(U)$, so $D(A)=$ $H_{0}^{1}(U) \cap H^{2}(U)$. In the following, we convert problem (1)-(3) into a random system without noise terms. Identify $\omega(t)$ with $W(t)$, i.e., $\omega(t)=W(t), t \in R$, and let $z\left(\theta_{t} \omega\right):=$ $-\int_{-\infty}^{0} e^{s}\left(\theta_{t} \omega\right)(s) d s(t \in R)$ be a Ornstein-Uhlenbeck stationary process which solves the Itô equation $d z+z d x=d W(t)$.

Let $\varepsilon=\frac{\left(\alpha+\alpha_{1}\right) \lambda_{1}}{2\left(\alpha+\alpha_{2}\right)^{2}+3 \lambda_{1}}$. By the transformation

$$
\varphi_{1}=u, \quad \varphi_{2}=u_{t}+\varepsilon u-a h(x) z\left(\theta_{t} \omega\right),
$$

Equations (1)-(3) are equivalent to the following determined system with random parameters in $E$ :

$$
\begin{aligned}
& \frac{d \varphi_{1}}{d t}=\varphi_{2}-\varepsilon \varphi_{1}+a h(x) z\left(\theta_{t} \omega\right), \\
& \frac{d \varphi_{2}}{d t}=\Delta \varphi_{1}+\varepsilon(\alpha-\varepsilon) \varphi_{1}+(\varepsilon-\alpha) \varphi_{2}-q\left(\varphi_{1}\right)\left(\varphi_{2}-\varepsilon \varphi_{1}\right) \\
& -\left(q\left(\varphi_{1}\right)+\alpha-\varepsilon-1\right) a h(x) z\left(\theta_{t} \omega\right)-f\left(\varphi_{1}, x\right)+g(x, t), \\
& \left.\varphi_{1}(x, t)\right|_{\partial U}=0, \\
& \varphi_{1}(\tau, \tau, x)=u_{\tau}(x), \quad \varphi_{2}(\tau, \tau, x)=\nu_{\tau}(x)=u_{1 \tau}+\varepsilon u_{\tau}(x)-a h(x) z\left(\theta_{\tau} \omega\right) .
\end{aligned}
$$


Then (8)-(11) can also be rewritten as a vector form:

$$
\begin{aligned}
& \dot{\varphi}+L(\varphi)=G\left(\varphi, \theta_{t} \omega, t\right), \\
& \varphi(\tau, \omega)=\varphi_{\tau}(\omega)=\left(u_{\tau}, u_{1, \tau}+\varepsilon u_{\tau}-a h(x) z\left(\theta_{\tau} \omega\right)\right)^{T}, \quad \tau \in R, t \geq \tau,
\end{aligned}
$$

where

$$
\varphi=\left(\begin{array}{l}
\varphi_{1} \\
\varphi_{2}
\end{array}\right), \quad L=\left(\begin{array}{cc}
\varepsilon I & -I \\
A-\varepsilon(\alpha-\varepsilon) I & (\alpha-\varepsilon) I
\end{array}\right)
$$

and

$$
\begin{aligned}
& G\left(\varphi, \theta_{t} \omega, t\right) \\
& \quad=\left(\begin{array}{c}
a h(x) z\left(\theta_{t} \omega\right) \\
-q\left(\varphi_{1}\right)\left(\varphi_{2}-\varepsilon \varphi_{1}\right)-\left[q\left(\varphi_{1}\right)+\alpha-\varepsilon-1\right] a h(x) z\left(\theta_{t} \omega\right)-f(u, x)+g(x, t)
\end{array}\right) .
\end{aligned}
$$

It is known from [28] that $-L$ is the infinitesimal generator of a $C^{0}$-semigroup $e^{-L t}$ on E. By assumption $\left(H_{2}\right)$ and the embedding relation $H_{0}^{1}(U) \hookrightarrow L^{6}(U)$, the function $G\left(\varphi, \theta_{t} \omega, t\right): E \rightarrow E$ is Lipschitz with respect to $\varphi$ for $t$ in a bounded interval and $\omega \in \Omega$, continuous in $(\varphi, t)$, and measurable in $\omega$ w.r.t $\mathcal{F}$. Thus, by the classical semigroup theory on the local existence and the uniqueness of solutions of evolution differential equations in [25], we have the following theorem.

Theorem 1 Consider the initial value problem (12)-(13), if assumptions $\left(H_{1}\right)$ and $\left(H_{2}\right)$ hold, then for each $\tau \in R, \omega \in \Omega$ and any $\varphi_{\tau} \in E$, there exists $T>0$ such that Eqs. (12)-(13) have a unique mild function $\varphi(\cdot)=\varphi\left(\cdot, \tau, \omega, \varphi_{\tau}\right) \in C([\tau, \tau+T) ; E)$, where $\varphi\left(\tau, \tau, \omega, \varphi_{\tau}\right)=\varphi_{\tau}$ and $\varphi(t)$ satisfies the integral equation

$$
\begin{aligned}
\varphi\left(t, \tau, \omega, \varphi_{\tau}\right)= & e^{-L(t-\tau)} \varphi_{\tau}(\omega) \\
& +\int_{\tau}^{t} e^{-L(t-r)} G\left(\varphi\left(r, \tau, \omega, \varphi_{\tau}\right), \theta_{r} \omega, r\right) d r, \quad \forall t \geq \tau .
\end{aligned}
$$

System (12)-(13) generates a continuous random dynamical system over $R$ and $\left(\Omega, \mathcal{F}, P,\left(\theta_{t}\right)_{t \in R}\right)$

$$
\Phi: R^{+} \times R \times \Omega \times E \rightarrow E, \quad\left(t, \tau, \omega, \varphi_{\tau}\right) \rightarrow \Phi\left(t, \tau, \omega, \varphi_{\tau}\right),
$$

where

$$
\begin{aligned}
\Phi & \left(t, \tau, \omega, \varphi_{\tau}(\omega)\right) \\
& =\varphi\left(t+\tau, \tau, \theta_{-\tau} \omega, \varphi_{\tau}\left(\theta_{-\tau} \omega\right)\right) \\
& =\left(\begin{array}{c}
u\left(t+\tau, \tau, \theta_{-\tau} \omega, \varphi_{-\tau}\left(\theta_{-\tau} \omega\right)\right) \\
u_{t}\left(t+\tau, \tau, \theta_{-\tau} \omega, \varphi_{-\tau}\left(\theta_{-\tau} \omega\right)\right)+\varepsilon u\left(t+\tau, \tau, \theta_{-\tau} \omega, \varphi_{-\tau}\left(\theta_{-\tau} \omega\right)\right)-a h(x) z\left(\theta_{t} \omega\right)
\end{array}\right),
\end{aligned}
$$




$$
\begin{aligned}
\Phi & \left(0, \tau, \omega, \varphi_{\tau} \omega\right) \\
& =\varphi_{\tau}\left(\theta_{-\tau} \omega\right) \\
& =\left(\begin{array}{c}
u\left(\tau, \tau, \theta_{-\tau} \omega, \varphi_{\tau}\left(\theta_{-\tau} \omega\right)\right) \\
u_{t}\left(\tau, \tau, \theta_{-\tau} \omega, \varphi_{\tau}\left(\theta_{-\tau} \omega\right)+\varepsilon u\left(\tau, \tau, \theta_{-\tau} \omega, \varphi_{\tau}\left(\theta_{-\tau} \omega\right)-a h(x) z(\omega)\right.\right.
\end{array}\right),
\end{aligned}
$$

and

$$
\Phi\left(t, \tau-t, \theta_{-t} \omega, \varphi_{\tau-t}\left(\theta_{-t} \omega\right)\right)=\varphi\left(\tau, \tau-t, \theta_{-\tau} \omega, \varphi_{\tau-t}\left(\theta_{-\tau} \omega\right)\right)
$$

So we have $\Gamma\left(t, \tau, \omega, Z_{\tau}\right)=R_{\varepsilon, \theta_{t} \omega}^{-1} \Phi\left(t, \tau, \omega, \varphi_{\tau}\right) R_{\varepsilon, \theta_{t} \omega}: Z_{\tau} \rightarrow Z\left(t+\tau, \tau, \theta_{-\tau} \omega, Z_{\tau}\right)$. Next, we use the transformation

$$
\psi_{1}=u, \quad \psi_{2}=u_{t}+\varepsilon u
$$

By using

$$
\psi=\left(\begin{array}{c}
\psi_{1} \\
\psi_{2}
\end{array}\right), \quad \tilde{G}(\psi)=\left(\begin{array}{c}
0 \\
g(x, t)-f(u, x)+a h(x) z\left(\theta_{t} \omega\right)
\end{array}\right)
$$

and

$$
H(\psi)=\left(\begin{array}{c}
\varepsilon \psi_{1}-\psi_{2} \\
A \psi_{1}-\varepsilon(\alpha-\varepsilon) \psi_{1}+(\alpha-\varepsilon) \psi_{2}+q\left(\psi_{1}\right)\left(\psi_{2}-\varepsilon \psi_{1}\right)
\end{array}\right),
$$

Eqs. (1)-(3) can be rewritten as

$$
\dot{\psi}+H(\psi)=\tilde{G}(\psi), \quad \psi_{\tau}(\omega)=\left(u_{\tau}, u_{1, \tau}+\varepsilon u_{\tau}\right)^{T}
$$

Thus

$$
\Psi\left(t, \tau, \omega, \psi_{\tau}\right)=T_{\varepsilon} \Gamma\left(t, \tau, \omega, Z_{\tau}\right) T_{-\varepsilon}: \psi_{\tau} \rightarrow \varphi\left(t+\tau, \tau, \theta_{-\tau} \omega, \psi_{\tau}\right),
$$

where

$$
\begin{aligned}
\Psi\left(t, \tau, \omega, \psi_{\tau}\right) & =\psi\left(t+\tau, \tau, \theta_{-\tau} \omega, \psi_{\tau}\right) \\
& =\varphi\left(t+\tau, \tau, \theta_{-\tau} \omega, \varphi_{\tau}\left(\theta_{-\tau} \omega\right)\right)+\left(0, a h(x) z\left(\theta_{t} \omega\right)\right)^{T} .
\end{aligned}
$$

Since $R_{\varepsilon, \theta_{t} \omega}:(a, b)^{T} \rightarrow\left(a, b+\varepsilon a-a h(x) z\left(\theta_{t} \omega\right)\right)^{T}$ is an isomorphism of $E$, then $\Phi, \Gamma, \Psi$ are equivalent to each other in dynamics.

Therefore, the existence of random attractors in any of these stochastic dynamical systems means that random attractors also exist in other dynamical systems. We will consider the existence of a random attractor for $R D S \Phi$ in the following. 


\section{Pullback absorbing set}

Let $\varphi=\left(\varphi_{1}, \varphi_{2}\right)^{T}$ be a solution of system (12)-(13). Rewriting system (12)-(13) as

$$
\dot{\varphi}+Q \varphi=\bar{G}\left(\varphi, \theta_{t} \omega\right), \quad \varphi(-\tau, \omega)=\left(u_{0}, u_{1}+\varepsilon u_{0}-a h(x) z\left(\theta_{-\tau} \omega\right)\right)^{T},
$$

where

$$
Q \varphi=\left(\begin{array}{c}
\varepsilon \varphi_{1}-\varphi_{2} \\
A \varphi_{1}-\varepsilon(\alpha-\varepsilon) \varphi_{1}+(\alpha-\varepsilon) \varphi_{2}+q\left(\varphi_{1}\right)\left(\varphi_{2}-\varepsilon \varphi_{1}\right)
\end{array}\right)
$$

and

$$
\bar{G}\left(\varphi, \theta_{t} \omega\right)=\left(\begin{array}{c}
a h(x) z\left(\theta_{t} \omega\right) \\
-\left(q\left(\varphi_{1}\right)+\alpha-\varepsilon-1\right) a h(x) z\left(\theta_{t} \omega\right)-f\left(\varphi_{1}, x\right)+g(x, t)
\end{array}\right),
$$

we have the following lemmas.

Lemma $1([26,27])$ For any $\varphi=\left(\varphi_{1}, \varphi_{2}\right)^{T} \in E,(Q \varphi, \varphi)_{E} \geq \frac{\varepsilon}{2}\|\varphi\|_{E}^{2}+\frac{\varepsilon}{4}\left\|\varphi_{1}\right\|_{H_{1}^{0}}+\frac{\alpha+\alpha_{1}}{2}\left\|\varphi_{2}\right\|_{L^{2}}^{2}$.

Lemma 2 If assumptions $\left(H_{1}\right)-\left(H_{2}\right)$ hold, then for any $\tau \in R, \omega \in \Omega$, there exists a tempered variable $M_{0}(\omega)$ (independent of $\tau$ ) such that, for any set $B \in D(E)$ and $\varphi_{\tau-t}\left(\theta_{-\tau} \omega\right) \in$ $B\left(\tau-t, \theta_{-t} \omega\right)$, there exists $T=T(\tau, \omega, B) \geq 0$ such that, for $t \geq T$, the solution $\varphi(\tau, \tau-t$, $\left.\theta_{-\tau} \omega, \varphi_{\tau-t}\left(\theta_{-\tau} \omega\right)\right) \in E$ of $(12)-(13)$ satisfies

$$
\left\|\varphi\left(\tau, \tau-t, \theta_{-\tau} \omega, \varphi_{\tau-t}\left(\theta_{-\tau} \omega\right)\right)\right\|_{E}^{2} \leq M_{0}^{2}(\omega), \quad \forall t \geq T(\tau, \omega, B) .
$$

Proof For any $\tau \in R, \omega \in \Omega$, let $\varphi(r)=\varphi\left(r, \tau-t, \theta_{\tau-t} \omega, \varphi_{\tau-t}\left(\theta_{-\tau} \omega\right)\right)=\left(\varphi_{1}, \varphi_{2}\right)^{T} \in E(r>\tau-t)$ be a solution of (12)-(13) with

$$
\varphi(\tau-t)=\varphi_{\tau-t}\left(\theta_{-\tau} \omega\right)=\left(u_{\tau-t}, u_{1, \tau-t}+\varepsilon u_{\tau-t}-a h(x) z\left(\theta_{-\tau} \omega\right)\right)^{T} \in E .
$$

Taking the inner product $(\cdot, \cdot)_{E}$ of (12) with $\varphi(r)$, according to Lemma 1, we have

$$
\frac{1}{2} \frac{d}{d t}\|\varphi\|_{E}^{2}+\frac{\varepsilon}{2}\|\varphi\|_{E}^{2}+\frac{\varepsilon}{4}\left\|\varphi_{1}\right\|_{H_{1}^{0}}^{2}+\frac{\alpha+\alpha_{1}}{2}\left\|\varphi_{2}\right\|_{L^{2}}^{2} \leq\left(\bar{G}\left(\varphi, \theta_{r-\tau} \omega, t\right), \varphi\right)_{E}
$$

and

$$
\begin{aligned}
& \left(\bar{G}\left(\varphi, \theta_{r-\tau} \omega, t\right), \varphi\right)_{E} \\
& \quad=\left(\left(a h(x) z\left(\theta_{r-\tau} \omega\right), \varphi_{1}\right)\right)-\left(\left(\alpha-\varepsilon-1+q\left(\varphi_{1}\right)\right) a h(x) z\left(\theta_{r-\tau} \omega\right), \varphi_{2}\right) \\
& \quad+\left(g(x, r), \varphi_{2}\right)-\left(f(u, x), \varphi_{2}\right) .
\end{aligned}
$$

By some simple computations, we obtain

$$
\begin{aligned}
& \left(\left(a h(x) z\left(\theta_{r-\tau} \omega\right), \varphi_{1}\right)\right) \leq \frac{1}{\sigma}\left|a z\left(\theta_{r-\tau} \omega\right)\right|^{2}\|h(x)\|_{1}^{2}+\frac{\sigma}{4}\left\|\varphi_{1}\right\|_{1}^{2}, \\
& \left(-q\left(\varphi_{1}\right) a h(x) z\left(\theta_{r-\tau}\right), \varphi_{2}\right) \\
& \quad=\delta\left\|\varphi_{2}\right\|_{L^{2}}^{2}+C_{\delta}\left(\alpha+\alpha_{2}\right)^{2}|a|^{2}\|h(x)\|_{L^{2}}^{2}\left|z\left(\theta_{r-\tau} \omega\right)\right|^{2}
\end{aligned}
$$


and

$$
\begin{aligned}
& \left(-(\alpha-\varepsilon-1) a h(x) z\left(\theta_{r-t} \omega\right), \varphi_{2}\right) \\
& \quad \leq \delta\left\|\varphi_{2}\right\|_{L^{2}}^{2}+C_{\delta}(\alpha-\varepsilon)^{2} a^{2}\|h(x)\|^{2}\left|z\left(\theta_{r-\tau} \omega\right)\right|^{2}, \\
& \left(g(x, r), \varphi_{2}\right) \leq \frac{1}{\alpha}\|g\|^{2}+\frac{\alpha}{2}\left\|\varphi_{2}\right\|^{2},
\end{aligned}
$$

where $\|g\|^{2}=\sup _{r \in R}\|g(\cdot, r)\|^{2}<\infty, \varphi_{2}=u_{t}+\varepsilon u-a h(x) z\left(\theta_{t} \omega\right)$.

By (5)-(7), we have

$$
\begin{aligned}
\left(f(u, x), \varphi_{2}\right)= & \left(f(u, x), u_{t}+\varepsilon u-a h(x) z\left(\theta_{r-\tau} \omega\right)\right) \\
= & \frac{d}{d t} \int_{U} F(u(r, x), x) d x+\varepsilon(f(u, x), u) \\
& -\left(f(u, x), a h(x) z\left(\theta_{r-\tau} \omega\right)\right) .
\end{aligned}
$$

From assumption $\left(\mathrm{H}_{2}\right)$, it is clear that

$$
(f(u, x), u)=\int_{U} f(u, x) u d x \geq \frac{1}{c_{1}}\left(\int_{U} F(u(r, x), x) d x-\int_{U} \phi_{2} d x\right) .
$$

With $u^{4} \leq \frac{1}{c_{2}} F(u, x)+\phi_{1}$ and $|f(u, x)| \leq c_{3}\left(1+u^{4}\right)$, we get

$$
\begin{aligned}
& \left(f(u, x), a h(x) z\left(\theta_{r-\tau} \omega\right)\right) \\
& \leq c_{4}|a|\|h(x)\|\left|z\left(\theta_{r-\tau} \omega\right)\right|+c_{5}|a|\left(\int_{U}|u|^{4} d x\right)^{\frac{3}{4}}\|h(x)\|_{L^{4}}\left|z\left(\theta_{r-\tau} \omega\right)\right| \\
& \leq c_{4}|a|\|h\|\left|z\left(\theta_{r-\tau} \omega\right)\right|+\frac{\varepsilon}{2 c_{2}} \bar{F}(u, x) \\
& \quad+c_{6} \int_{U} \phi_{1} d x+c_{7} a^{4}\|h(x)\|_{1}^{4}\left|z\left(\theta_{r-\tau} \omega\right)\right|^{4},
\end{aligned}
$$

here $\bar{F}(u, x)=\int_{U} F(u, x) d x$. By taking (26)-(33) into (25), we have

$$
\begin{aligned}
\frac{1}{2} \frac{d}{d t}[ & \left.\|\varphi\|^{2}+2 \bar{F}(u, x)\right]+\frac{1}{2}\|\varphi\|_{E}^{2}+\frac{\varepsilon}{4}\left\|\varphi_{1}\right\|_{H_{0}^{1}}^{2}+\frac{\alpha+\alpha_{2}}{2}\left\|\varphi_{2}\right\|_{L^{2}}^{2} \\
& +\frac{\varepsilon}{c_{1}}\left[\bar{F}(u, x)-2 \int_{U} \phi_{2}(x) d x\right]+c_{4}|a|\|h(x)\|\left|z\left(\theta_{r-\tau} \omega\right)\right|+\frac{\varepsilon}{2 c_{2}} \bar{F}(u, x) \\
& +c_{6} \int_{U} \phi_{1}(x) d x+c_{7} a^{4}\|h(x)\|_{1}^{4}\left|z\left(\theta_{r-\tau} \omega\right)\right|^{4} \\
\leq & \frac{1}{\varepsilon} a^{2}\left|z\left(\theta_{r-\tau} \omega\right)\right|^{2}\|h(x)\|_{1}^{2}+\frac{\varepsilon}{4}\|u\|_{1}^{2}+\frac{1}{\alpha}\left[\|g\|^{2}+a^{2}\left|z\left(\theta_{r-\tau} \omega\right)\right|^{2}\|h(x)\|^{2}\right] \\
& +\frac{\alpha}{2}\left\|\varphi_{2}\right\|^{2}+\delta\left\|\varphi_{2}\right\|_{L^{2}}^{2}+C_{\delta}\left(2 \alpha+\alpha_{2}\right)^{2} a^{2}\|h(x)\|_{L^{2}}^{2}\left|z\left(\theta_{r-\tau} \omega\right)\right|^{2} .
\end{aligned}
$$


Let

$$
\begin{aligned}
\beta\left(\theta_{r-\tau} \omega\right)= & -\frac{2 \varepsilon}{c_{1}} \int_{U} \phi_{2}(x) d x+c_{4}|a|\|h(x)\|\left|z\left(\theta_{r-\tau} \omega\right)\right| \\
& +c_{6} \int_{U} \phi_{1}(x) d x+c_{7} a^{4}\|h(x)\|_{1}^{4}\left|z\left(\theta_{r-\tau} \omega\right)\right|^{4} \\
& +\frac{1}{\alpha}\left[\|g\|^{2}+a^{2}\left|z\left(\theta_{r-\tau} \omega\right)\right|^{2}\|h(x)\|^{2}\right] \\
& +C_{\delta}\left(2 \alpha+\alpha_{2}\right)^{2} a^{2}\|h(x)\|_{L^{2}}^{2}\left|z\left(\theta_{r-\tau} \omega\right)\right|^{2} \\
= & c_{8}+c_{9} a^{4}\left|z\left(\theta_{r-\tau} \omega\right)\right|^{4} .
\end{aligned}
$$

By choosing $\delta$ small enough, we get

$$
\frac{d}{d t} y(r)+\rho y(r) \leq \beta\left(\theta_{r-\tau}, \omega\right), \quad \forall r \geq \tau-t,
$$

where $y(r)=\|\varphi(r)\|_{E}^{2}+2 \bar{F}(u, x)$ and $\rho=\min \left\{\frac{\varepsilon+2}{4}, \frac{\alpha+\alpha_{2}+2}{2}, \frac{2 \varepsilon}{c_{1}}\right\}$. By Gronwall's inequality to Eq. (36), we have

$$
\begin{aligned}
& y(r, \tau\left.-t, \theta_{-\tau} \omega, \varphi_{\tau-t}\left(\theta_{-\tau} \omega\right)\right) \\
& \leq y\left(\tau-t, \tau-t, \theta_{-\tau} \omega, \varphi_{\tau-t}\left(\theta_{-\tau} \omega\right)\right) e^{-\rho(r+t-\tau)} \\
& \quad+\int_{\tau-t}^{r} \beta\left(\theta_{s-\tau} \omega\right) e^{-\rho(r-s)} d s
\end{aligned}
$$

where

$$
\begin{aligned}
& y\left(\tau-t, \tau-t, \theta_{-\tau} \omega, \varphi_{\tau-t}\left(\theta_{-\tau} \omega\right)\right) \\
& \quad=\left\|\varphi_{\tau-t}\left(\theta_{-\tau} \omega\right)\right\|_{E}^{2}+2 \int_{U} F(u(\tau-t, x), x) d x \\
& \leq\left\|\varphi_{\tau-t}\left(\theta_{-\tau} \omega\right)\right\|_{E}^{2}+2 c_{10}\left(|U|+\left\|u_{\tau-t}\right\|_{1}^{4}+2 \int_{U} \phi_{2}(x) d x\right), \\
& \int_{\tau-t}^{r} \beta\left(\theta_{s-\tau}\right) e^{-\sigma(r-s)} d s=\frac{c_{8}}{\sigma}+c_{9} a^{4} \int_{\tau-t}^{r}\left|z\left(\theta_{s-r} \omega\right)\right|^{4} e^{-\sigma(r-s)}
\end{aligned}
$$

and

$$
\begin{aligned}
& y\left(r, \tau-t, \theta_{-\tau} \omega, \varphi_{\tau-t}\left(\theta_{-\tau} \omega\right)\right) \\
& \quad \geq\left\|\varphi\left(r, \tau-t, \theta_{-\tau} \omega, \varphi_{\tau-t}\left(\theta_{-\tau} \omega\right)\right)\right\|_{E}^{2}-2 \int_{U} \phi_{1}(x) d x .
\end{aligned}
$$

Thus by (37)-(40), for $r \geq \tau-t$, we have

$$
\begin{aligned}
& \left\|\varphi\left(r, \tau-t, \theta_{-\tau} \omega, \varphi_{\tau-t}\left(\theta_{-\tau} \omega\right)\right)\right\|_{E}^{2} \\
& \quad \leq y(r)+2 \int_{U} \phi_{1}(x) d x
\end{aligned}
$$




$$
\begin{aligned}
& \leq\left(\left\|\varphi_{\tau-t}\left(\theta_{-\tau} \omega\right)\right\|_{E}^{2}+2 c_{10}\left(|U|+\left\|u_{\tau-t}\right\|_{1}^{4}+2 \int_{U} \phi_{2}(x) d x\right)\right) e^{-\sigma(r+t-\tau)} \\
& \quad+2 \int_{U} \phi_{1}(x) d x+c_{11}+c_{12} a^{4} \int_{\tau-t}^{r}\left|z\left(\theta_{s-r} \omega\right)\right|^{4} e^{-\sigma(r-s)} d s .
\end{aligned}
$$

Therefore

$$
\begin{aligned}
& \left\|\varphi\left(r, \tau-t, \theta_{-\tau} \omega\right), \varphi_{\tau-t}\left(\theta_{-\tau} \omega\right)\right\|_{E}^{2} \\
& \leq\left(\left\|\varphi_{\tau-t}\left(\theta_{-\tau} \omega\right)\right\|_{E}^{2}+2 c_{10}\left(|U|+\left\|u_{\tau-t}\right\|_{1}^{4}+2 \int_{U} \phi_{2}(x) d x\right)\right) e^{-\sigma t} \\
& \quad+2 \int_{U} \phi_{1}(x) d x+c_{11}+c_{12} a^{4} \int_{\tau-t}^{r}\left|z\left(\theta_{s-r} \omega\right)\right|^{4} e^{-\sigma t} d s .
\end{aligned}
$$

For any set $B(\tau, \omega) \in B \in D(E)$,

$$
\begin{aligned}
& \varphi_{\tau-t}\left(\theta_{-\tau} \omega\right) \\
& \quad=\left(u_{\tau-t}, u_{1, \tau-t}+\varepsilon u_{\tau-t}-a h(x) z\left(\theta_{-t} \omega\right)\right)^{T} \\
& \quad \in B\left(\tau-t, \theta_{-t} \omega\right) \in D(E) .
\end{aligned}
$$

We have

$$
\begin{aligned}
& \lim \sup _{t \rightarrow+\infty}\left(\left\|\varphi_{\tau-t}\left(\theta_{-\tau} \omega\right)\right\|_{E}^{2}\right. \\
& \left.\quad+2 c_{10}\left(|U|+\left\|u_{\tau-t}\right\|_{1}^{4}+2 \int_{U} \phi_{2}(x) d x\right)\right) e^{-\rho t}=0 .
\end{aligned}
$$

Taking

$$
M_{0}^{2}(\omega)=2 c_{11}+2 c_{12} a^{4} \int_{-\infty}^{0}\left|z\left(\theta_{s} \omega\right)\right|^{4} e^{\rho s} d s<\infty
$$

which is a tempered random variable, $B_{0}(\omega)=\left\{\varphi \in E:\|\varphi\|_{E} \leq M_{0}(\omega)\right\}$ is a close measurable absorbing ball in $D(E)$, and there exists $T(\tau, \omega, B) \geq 0$ for all $t \geq T(\tau, \omega, B)$ such that

$$
\varphi\left(\tau, \tau-t, \theta_{-\tau} \omega, \varphi_{\tau-t}\left(\theta_{-\tau} \omega\right)\right) \in B_{0}(\omega)
$$

$B_{0}(\omega)$ is the random absorbing set for $\Phi$. The proof is completed.

\section{Decomposition of the equations}

In this section, for proving asymptotic compactness of the random dynamical system $\Phi$, we decompose the solution of Eq. (12)-(13) with different initial data into a sum of two parts, one part decays exponentially and another one is bounded in a higher regular space by using the method in $[4,13]$.

For any $\tau \in R$ and $\omega \in \Omega$, assume that

$$
B_{1}(\tau, \omega)=\bigcup_{t \geq T\left(\tau, \omega, B_{0}\right)} \varphi\left(\tau, \tau-t, \theta_{-t} \omega, B_{0}\left(\theta_{-t} \omega\right)\right) \subseteq B_{0}(\omega)
$$


Let $\varphi(r)=\varphi\left(r, \tau-t, \theta_{-\tau} \omega, \varphi_{\tau-t}\left(\theta_{-\tau} \omega\right)\right)$ be a solution of system (12)-(13), with $\varphi_{\tau-t}\left(\theta_{-\tau} \omega\right) \in$ $B_{1}\left(\tau-t, \theta_{-t} \omega\right) \subseteq B_{0}\left(\theta_{-t} \omega\right)$. Thus $\varphi(r) \in B_{0}\left(\theta_{r-\tau} \omega\right)$ for all $r \geq \tau-t$. We decompose $\varphi(r)$ into

$$
\varphi(r)=\varphi_{L}(r)+\varphi_{N}(r), \quad \varphi_{L}(r)=\left(u_{L}, v_{L}\right)^{T}, \varphi_{N}(r)=\left(u_{N}, \varphi_{2 N}\right)^{T},
$$

where $\varphi_{L}(r)$ and $\varphi_{N}(r)$ satisfy

$$
\left\{\begin{array}{l}
\dot{\varphi}_{L}+Q \varphi_{L}+\left(\begin{array}{c}
0 \\
f_{1}\left(u_{L}, x\right)
\end{array}\right)=0 \\
\varphi_{L}\left(r, \tau-t, \theta_{-\tau} \omega, \varphi_{\tau-t}\left(\theta_{-\tau} \omega\right)\right)=\varphi_{L, \tau-t}=\left(u_{\tau-t}, u_{1, \tau-t}+\varepsilon u_{\tau-t}\right)^{T}
\end{array}\right.
$$

and

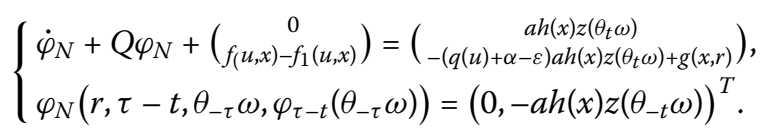

First, let us estimate the component $\varphi_{L}$ which decays exponentially.

Lemma 3 Under assumptions $\left(H_{1}\right)-\left(H_{2}\right)$, for any $\tau \in R, \omega \in \Omega, t \geq 0, r \geq \tau-t$, and $\varphi_{\tau-t}\left(\theta_{-\tau} \omega\right) \in B_{0}\left(\theta_{-t} \omega\right)$, the solution $\varphi_{L}(r)=\varphi_{L}\left(r, \tau-t, \varphi_{L, \tau-t}\right)$ of $(46)$ satisfies that

$$
\left\|\varphi_{L}\left(r, \tau-t, \varphi_{L, \tau-t}\right)\right\|_{E}^{2} \leq M_{L}^{2} e^{-2 \sigma_{1}(t+r-\tau)}
$$

holds.

Proof Let $\varphi_{L}=\left(\varphi_{L, 1}, \varphi_{L, 2}\right)=\left(u_{L}, v_{L}\right)=\left(u_{L}, u_{L, t}+\varepsilon u\right)$. Taking the inner product $(\cdot, \cdot)_{E}$ of Eq. (46) with $\varphi_{L}(r)$, we have

$$
\frac{1}{2} \frac{d}{d t}\left\|\varphi_{L}(r)\right\|_{E}^{2}+2\left(Q \varphi_{L}, \varphi_{L}\right)_{E}=\left(\left(\begin{array}{c}
0, \\
-f_{1}\left(u_{L}, x\right)
\end{array}\right), \varphi_{L}\right),
$$

where $\left(\left(\begin{array}{c}0, \\ -f_{1}\left(u_{L}, x\right)\end{array}\right), \varphi_{L}\right)=-\frac{d}{d t} \int_{U} F\left(\varphi_{L 1}, x\right)-\varepsilon \int_{U} f\left(\varphi_{L 1}, x\right) \varphi_{L 1}$. By Lemma 1 , we see that

$$
2\left(Q\left(\varphi_{L}\right), \varphi_{L}\right) \geq \varepsilon\left\|\varphi_{L}\right\|_{E}^{2}+\frac{\varepsilon}{2}\left\|\varphi_{L, 1}\right\|_{H_{0}^{2}}^{2}+\left(\alpha+\alpha_{1}\right)\left\|\varphi_{L, 2}\right\|_{L^{2}}^{2} .
$$

By assumption $\left(H_{2}\right)$, we obtain

$$
\left.f\left(\varphi_{L 1}, x\right), \varphi_{L 1}\right)=(f(u, x), u) \geq \frac{1}{C_{4}} \bar{F}_{1}\left(u_{L}, x\right)-\varepsilon \int_{U} \phi_{2}(x) d x .
$$

Thus, by Eqs. (49)-(51), we have

$$
\begin{aligned}
& \frac{d}{d t}\left[\left\|\varphi_{L}\right\|_{E}^{2}+2 \bar{F}_{1}\left(u_{L}, x\right)\right]+\varepsilon\left\|\varphi_{L}\right\|_{E}^{2}+\frac{\varepsilon}{2}\left\|\varphi_{L, 1}\right\|_{H_{0}^{2}}^{2} \\
& \quad+\left(\alpha+\alpha_{1} \| \varphi_{L, 2}\right) \|_{L^{2}}^{2}+\frac{2 \varepsilon}{C_{4}} \bar{F}_{1}\left(u_{L}, x\right) \\
& \leq 2 \varepsilon \int_{U} \phi_{2}(x) d x .
\end{aligned}
$$


Hence we can conclude that there exists $\sigma_{L}=\min \left\{\frac{2 \varepsilon}{C_{4}}, \frac{\varepsilon}{2}, \frac{\alpha+\alpha_{2}}{2}\right\}$ such that

$$
\frac{d}{d t} y_{L}+\sigma_{L} y_{L}(r) \leq \beta_{L}
$$

where $y_{L}(r)=\left\|\varphi_{L}(r)\right\|_{E}^{2}+2 \bar{F}_{L}\left(u_{L}, x\right) \geq 0, \beta_{L}=2 \varepsilon \int_{U} \phi_{2}(x) d x$. Since $\varphi_{\tau-t}\left(\theta_{-\tau} \omega\right)+$ $\left(0, a h(x) z\left(\theta_{-t} \omega\right)\right)^{T} \in B_{0}\left(\theta_{-t} \omega\right)$, we have

$$
\left\|\varphi_{L, \tau-t}\right\|_{E} \leq M_{0}\left(\theta_{-t} \omega\right)+|a|\|h(x)\| \cdot\left|z\left(\theta_{-t} \omega\right)\right|
$$

Notice that $\varphi_{L, \tau-t}$ is independent of $\omega$, so replacing $\omega$ by $\theta_{-t} \omega$, then

$$
\left\|\varphi_{L, \tau-t}\right\|_{E} \leq M_{0}(\omega)+|a|\|h(x)\| \cdot|z(\omega)|
$$

Applying Gronwall's inequality to Eq. (53), we have

$$
\begin{aligned}
\left\|\varphi_{L}\left(r, \tau-t, \varphi_{L, \tau-t}\right)\right\|_{E}^{2} & \leq y_{L}\left(r, \tau-t, \varphi_{L, \tau-t}\right) \\
& \leq y\left(\tau-t, \tau-t, \varphi_{L, \tau-t}\right) e^{-\sigma_{L}(t+r-\tau)}+\frac{\beta_{L}}{\sigma_{L}} \\
& \leq\left(\left\|\varphi_{L, \tau-t}\right\|_{E}^{2}+c_{10}\left(|U|+\left\|u_{\tau-t}\right\|_{1}^{4}+\int_{U} \phi_{2} d x\right)\right) e^{-\sigma_{L}(t+r-\tau)}+\frac{\beta_{L}}{\sigma_{L}} \\
& =M_{1}^{2}(\omega) .
\end{aligned}
$$

Next, we consider Eq. (49). Due to $\left(f_{1}\left(u_{L}, x\right), u_{L}\right) \geq 0,\left|f_{1}\left(u_{L}(r), x\right)\right| \leq c_{13}\left(\left|u_{L}(r)\right|^{3}+\left|u_{L}(r)\right|\right)$ and assumption $\left(H_{2}\right)$, according to Sobolev embedding $H_{0}^{1}(U) \subset L^{4}(U) \subset L^{2}(U)$, there exists $M_{2}(\omega)>0$ such that

$$
0 \leq \bar{F}_{1}\left(u_{L}(r), x\right) \leq c_{14}\left(\left\|u_{L}(r)\right\|_{L^{4}}^{4}+\left\|u_{L}(r)\right\|^{2}\right) \leq M_{2}(\omega)\left\|u_{L}(r)\right\|_{1}^{2}
$$

That is, $\left\|u_{L}(r)\right\|_{1}^{2} \geq \frac{1}{M_{2}(\omega)} \bar{F}_{1}\left(u_{L}(r), x\right)$. From (49)-(50) and (55), for any $r \geq \tau-t$, we have

$$
\frac{d}{d t}\left[\left\|\varphi_{L}\right\|_{E}^{2}+2 \bar{F}_{1}\left(u_{L}, x\right)\right]+\frac{\varepsilon}{2}\left\|\varphi_{L}\right\|_{E}^{2}+\frac{\varepsilon}{2 M_{2}(\omega)} \bar{F}_{1}\left(u_{L}, x\right) \leq 0 .
$$

So the inequality

$$
\frac{d}{d t}\left[\left\|\varphi_{L}\right\|_{E}^{2}+2 \bar{F}_{1}\left(u_{L}, x\right)\right]+2 \sigma_{1}(\omega)\left[\left\|\varphi_{L}\right\|_{E}^{2}+2 \bar{F}_{1}\left(u_{L}, x\right)\right] \leq 0
$$

holds, where $\sigma_{1}=\min \left\{\frac{\varepsilon}{4}, \frac{\varepsilon}{8 M_{2}(\omega)}\right\}>0$. Thus, we get

$$
\begin{aligned}
\left\|\varphi_{L}\left(r, \tau-t, \varphi_{L, \tau-t}\right)\right\|_{E}^{2} & \leq\left[\left\|\varphi_{L, \tau-t}\right\|_{E}^{2}+2 \bar{F}_{1}\left(u_{L, \tau-t}, x\right)\right] e^{-2 \sigma_{1}(r+t-\tau)} \\
& \leq\left(\left\|\varphi_{L, \tau-t}\right\|_{E}^{2}+c_{10}\left(|U|+\left\|u_{\tau-t}\right\|_{1}^{4}+\int_{U} \phi_{2} d x\right)\right) e^{-2 \sigma_{1}(t+r-\tau)} \\
& =M_{L}^{2} e^{-2 \sigma_{1}(r+t-\tau)}
\end{aligned}
$$

The proof is completed. 
For the component $\varphi_{N}$, which is ultimately pullback bounded in a higher regular space, we have the following estimate.

Lemma 4 If assumptions $\left(H_{1}\right)-\left(H_{2}\right)$ hold, then for any $\tau \in R, \omega \in \Omega$, and $t \geq 0$, there exist a positive constant $v \in\left(0, \min \left\{\frac{1}{4}, \frac{3-p}{4}\right\}\right)$ and a positive-value random variable $M_{N}(\omega)>0$ such that the solution $\varphi_{N}(r)=\left(\varphi_{1 N}(r), \varphi_{2 N}(r)\right)^{T}$ of Eq. (47) satisfies the following:

$$
\left\|A^{v} \varphi_{N}\left(r, \tau-t, \theta_{-\tau} \omega, \varphi_{\tau-t}\left(\theta_{-\tau} \omega\right)\right)\right\|_{E}^{2} \leq M_{N}^{2}(\omega)
$$

for $t \geq 0$ and $\varphi_{\tau-t}\left(\theta_{-\tau} \omega\right) \in B_{0}\left(\theta_{-t} \omega\right)$.

Proof Taking the inner product of Eq. (47) in $E$ with

$$
A^{2 v} \varphi_{N}=\left(A^{2 v} \varphi_{1 N}, A^{2 v} \varphi_{2 N}\right)=\left(A^{2 v} u_{N}, A^{2 v} \varphi_{2 N}\right),
$$

we have

$$
\begin{aligned}
& \frac{1}{2} \frac{d}{d t}\left[A^{v}\left\|\varphi_{N}\right\|^{2}+2 \int_{U}\left[f(u, x)-f_{1}\left(u_{L}, x\right)\right] A^{2 v} u_{N} d x\right]+\left(Q\left(\varphi_{N}, A^{2 v}\right)\right) \\
& \quad+\varepsilon \int_{U}\left[f(u, x)-f_{1}\left(u_{L}, x\right)\right] A^{2 v} u_{N} d x \\
& \quad-\int_{U}\left(\left[f_{1, u}^{\prime}(u, x)-f_{1, u}^{\prime}\left(u_{L}, x\right)\right] u_{t}+f_{1, u}^{\prime}\left(u_{L}, x\right) u_{N t}+f_{2, u}^{\prime}(u, x) u_{t}\right) A^{2 v} u_{N} d x \\
& =\left(a h(x) z\left(\theta_{r-\tau} \omega\right), A^{2 v} u_{N}\right)_{1}-\left(f(u, x)-f_{1}\left(u_{L}, x\right), A^{2 v} a h(x) z\left(\theta_{r-\tau} \omega\right)\right) \\
& \quad+\left(g(x, r)-(\alpha-\varepsilon-1) a h(x) z\left(\theta_{r-\tau} \omega\right), A^{2 v} \varphi_{2 N}\right) \\
& \quad+\left(-q\left(\varphi_{1 N}\right) a h(x) z\left(\theta_{r-\tau} \omega\right), A^{2 v} \varphi_{2 N}\right) .
\end{aligned}
$$

Similar to (50), we get

$$
\frac{\varepsilon}{2}\left\|A^{v} \varphi_{N}\right\|_{E}^{2}+\frac{\varepsilon}{4}\left\|A^{v} u_{N}\right\|_{H_{0}^{1}}^{2}+\frac{\alpha+\alpha_{1}}{2}\left\|A^{v} \varphi_{2 N}\right\|_{L^{2}}^{2} \leq\left(Q \varphi_{N}, A^{2 v} \varphi_{N}\right)_{E} .
$$

By some computations, we have

$$
\begin{aligned}
& \left(a h(x) z\left(\theta_{r-\tau} \omega\right), A^{2 v} u_{N}\right) \leq \frac{2 a^{2}}{\varepsilon} z^{2}\left(\theta_{r-\tau} \omega\right)\|h(x)\|_{1}^{2}+\frac{\varepsilon}{8}\left\|A^{v+\frac{1}{2}} u_{N}\right\|^{2}, \\
& \left(f(u, x)-f_{1}\left(u_{L}, x\right), A^{2 v} a h(x) z\left(\theta_{r-\tau} \omega\right)\right) \\
& \quad \leq K_{1}\left(r, \tau-t, \theta_{-t} \omega\right)+c_{15} a^{2}\|h(x)\|_{1}^{2}\left|z\left(\theta_{r-\tau} \omega\right)\right|^{2},
\end{aligned}
$$

and

$$
\begin{aligned}
& \left(g(x, r)-(\alpha-\varepsilon-1) a h(x) z\left(\theta_{r-\tau} \omega\right), A^{2 v} \varphi_{2 N}\right) \\
& \quad \leq \frac{2}{\alpha}\left[\|g\|_{1}^{2}+(\alpha-\varepsilon-1)^{2} a^{2}\|h(x)\|_{2}^{2} z^{2}\left(\theta_{r-\tau} \omega\right)\right]+\frac{\alpha}{4}\left\|A^{v} \varphi_{2 N}\right\|^{2},
\end{aligned}
$$


Yo and Chang Advances in Difference Equations

(2020) 2020:221

Page 14 of 19

where $\|g\|_{1}^{2}=\sup _{r \in R}\|g(\cdot, r)\|_{1}^{2}<\infty$. Thus, by taking (60)-(63) into (59), we obtain

$$
\begin{aligned}
\frac{1}{2} \frac{d}{d t} & \left(\left\|A^{v} \varphi_{N}\right\|_{E}^{2}+2 \int_{U}\left[f(u, x)-f_{1}\left(u_{L}, x\right)\right] A^{2 v} u_{N} d x\right)+\sigma_{2}\left\|A^{v} \varphi_{N}\right\|_{E}^{2} \\
& +\varepsilon \int_{U}\left[f(u, x)-f_{1}\left(u_{L}, x\right) \cdot A^{2 v} u_{N} d x\right]-\int_{U}\left(\left[f_{1, u}^{\prime}(u, x)\right.\right. \\
& \left.\left.-f_{1, u}^{\prime}\left(u_{L}, x\right)\right] u_{t}+f_{1, u}^{\prime}\left(u_{L}, x\right) u_{N, t}+f_{2, u}^{\prime}(u, x) u_{t}\right) \cdot A^{2 v} u_{N} d x \\
\leq & K_{2}\left(r, r-t, \theta_{-t} \omega\right)+c_{16} a^{2} z^{2}\left(\theta_{r-\tau} \omega\right) .
\end{aligned}
$$

Let $\sigma_{2}=\min \left\{\frac{\varepsilon}{4}, \frac{2 \varepsilon+\alpha}{4}\right\}$, then by Hölder's inequality, we have

$$
\begin{aligned}
& \left|\left(f_{1, u}^{\prime}\left(u_{L}, x\right) u_{N, t}, A^{2 v} u_{N}\right)\right| \\
& \quad \leq c_{17}\left(\int_{U}\left(1+u_{L}^{2}\right)^{3} d x\right)^{\frac{1}{3}}\left(\int_{U}\left|A^{2 v} u_{N}\right|^{\frac{6}{1+4 v}} d x\right)^{\frac{1+4 v}{6}}\left(\int_{u}\left|u_{N, t}\right|^{\frac{6}{3-4 v}} d x\right)^{\frac{3-4 v}{6}} \\
& \quad \leq c_{18}\left\|A^{v+\frac{1}{2}} u_{N}\right\| \cdot\left\|A^{v} u_{N, t}\right\|
\end{aligned}
$$

for $r>\tau-t$. We have

$$
\begin{aligned}
& \left|\left(f_{2, u}^{\prime}(u, x) u_{t}, A^{2 v} u_{N}\right)\right| \\
& \quad \leq c_{19} \int_{U}\left|u_{t}\right| \cdot\left(1+|u|^{p}\right) \cdot\left|A^{2 v} u_{N}\right| d x \\
& \quad \leq\left(\int_{U}\left|u_{t}\right|^{2} d x\right)^{\frac{1}{2}}\left(\int_{U}\left(1+|u|^{p}\right)^{\frac{6}{2-4 v}} d x\right)^{\frac{2-4 v}{6}}\left(\int_{U}\left|A^{2 v} u_{N}\right|^{\frac{6}{1+4 v}}\right)^{\frac{1+4 v}{6}} \\
& \quad \leq\left\|u_{t}\right\|_{0}\left(1+\|u\|_{1}^{p}\right)\left\|A^{v+\frac{1}{2}} u_{N}\right\| \leq K_{3}\left(r, \tau-t, \theta_{-t} \omega\right)\left\|A^{v+\frac{1}{2}} u_{N}\right\|
\end{aligned}
$$

and

$$
\begin{aligned}
& \left|\left(\left[f_{1, u}^{\prime}(u, x)-f_{1, u}^{\prime}\left(u_{L}, x\right)\right] u_{t}, A^{2 v} u_{N}\right)\right| \\
& \quad \leq \int_{U}\left|u_{t}\right|\left(1+\left|u_{N}\right|+\left|u_{L}\right|\right)\left|u_{N}\right|\left|A^{2 v} u_{N}\right| d x \\
& \leq\left(\int_{U}\left|u_{t}\right|^{2} d x\right)^{\frac{1}{2}}\left(\int_{u}\left(1+\left|u_{N}\right|+\left|u_{L}\right|\right)^{6} d x\right)^{\frac{1}{6}} \\
& \quad \times\left(\int_{U}\left|u_{N}\right|^{\frac{6}{3-4 v}} d x\right)^{\frac{3-4 v}{6}}\left(\int_{U}\left|A^{2 v} u_{N}\right|^{\frac{6}{1+4 v}} d x\right)^{\frac{1+4 v}{6}} \\
& \leq K_{4}\left(r, \tau-t, \theta_{-t} \omega\right)\left\|A^{v+\frac{1}{2}} u_{N}\right\| .
\end{aligned}
$$

By putting the above inequalities into (64), we get

$$
\begin{aligned}
& \frac{1}{2} \frac{d}{d t}\left(\left\|A^{v} \varphi_{N}\right\|_{E}^{2}+2 \int_{U}\left[f(u, x)-f_{1}\left(u_{L}, x\right)\right] A^{2 v} u_{N} d x\right) \\
& \quad+\sigma_{1}\left[\left\|A^{v} \varphi_{N}\right\|_{E}^{2}+2 \int_{U}\left[f(u, x)-f_{1}\left(u_{L}, x\right)\right] A^{2 v} u_{N} d x\right]
\end{aligned}
$$




$$
\begin{aligned}
\leq & c_{20}\left\|A^{v+\frac{1}{2}} u_{N}\right\|\left\|A^{v} u_{N, t}\right\|+K_{2}\left(r, \tau-t, \theta_{-t} \omega\right)+c_{16} a^{2} z^{2}\left(\theta_{r-\tau} \omega\right) \\
& +\left\|u_{t}\right\|_{0}\left(1+\|u\|_{1}^{p}\left\|A^{v+\frac{1}{2}} u_{N}\right\|\right)+K_{4}\left(r, \tau-t, \theta_{-t} \omega\right)\left\|A^{v+\frac{1}{2}} u_{N}\right\| .
\end{aligned}
$$

That is,

$$
\begin{aligned}
& \frac{d}{d t} y_{1}\left(r, \tau-t, \theta_{-t} \omega, \varphi_{\tau-t}\left(\theta_{-t} \omega\right)\right)+\sigma_{1} y_{1}\left(r, \tau-t, \theta_{-t} \omega, \varphi_{\tau-t}\left(\theta_{-t} \omega\right)\right) \\
& \quad \leq K_{5}\left(r, \tau-t, \theta_{-t} \omega\right)+c_{21} a^{2} z^{2}\left(\theta_{r-\tau} \omega\right)
\end{aligned}
$$

and

$$
\begin{aligned}
& y_{1}(r)=\left\|A^{v} \varphi_{N}\right\|_{E}^{2}+2 \int_{U}\left[f(u, x)-f_{1}\left(u_{L}, x\right)\right] A^{2 v} u_{N}, \\
& \beta_{2}\left(\theta_{r-\tau} \omega\right)=K_{5}\left(r, \tau-t, \theta_{-t} \omega\right)+c_{21} a^{2} z^{2}\left(\theta_{r-\tau} \omega\right) .
\end{aligned}
$$

It follows from (69) that

$$
\frac{d}{d t} y_{1}(r)+\sigma_{1} y_{1}(r) \leq \beta_{2}\left(\theta_{r-\tau} \omega\right), \quad \forall r \geq \tau-t
$$

Note that $y_{1}\left(\tau-t, \tau-t, \theta_{-\tau} \omega, \varphi_{\tau-t} \omega\right) \leq a^{2}\left\|A^{v} h(x)\right\|^{2} z^{2}\left(\theta_{-t} \omega\right)$, then by applying Gronwall's inequality to (72) on $[\tau-t, r](r \geq \tau-t)$, we have

$$
\begin{aligned}
& y_{1}\left(r, \tau-t, \theta_{-t} \omega, \varphi_{\tau-t} \omega\right) \\
& \quad \leq y_{1}\left(\tau-t, \tau-t, \theta_{-\tau} \omega, \varphi_{\tau-t}\left(\theta_{-\tau} \omega\right)\right)+\int_{\tau-t}^{r} \beta_{2}\left(\theta_{\tau-t} \omega\right) e^{r-\tau+t} \\
& \quad \leq c_{21}\|h(x)\|_{1}^{2} a^{2} z^{2}\left(\theta_{-t} \omega\right) e^{r-\tau+t}+\int_{\tau-t}^{r} \beta_{2}\left(\theta_{\tau-t} \omega\right) e^{r-\tau+t} \\
& \quad \leq a^{2} M_{8}(\omega)+M_{9}(\tau, \omega)
\end{aligned}
$$

for

$$
\begin{aligned}
& \left|\left[\int_{U} f(u, x)-f_{1}\left(u_{L}, x\right)\right]\right| A^{2 v} u_{N} d x \\
& \quad=\int_{u}\left[f_{2}(u, x)+f_{1}(u, x)-f_{1}\left(u_{L}, x\right)\right] A^{2 v} u_{N} d x,
\end{aligned}
$$

where

$$
\begin{aligned}
\left|\int_{U} f_{2}(u, x) A^{2 v} u_{N} d x\right| & \leq c_{31} \int_{U}\left(1+|u|^{p}\right) A^{2 v} u_{N} d x \\
& \leq c_{31}\left(\int_{U}\left(1+|u|^{p}\right)^{\frac{6}{5-4 v}}\right)^{\frac{5-4 v}{6}}\left(\int_{u}\left|A^{2 v} u_{N}\right|^{\frac{6}{1+4 v}}\right)^{\frac{1+4 v}{6}} \\
& \leq K_{8}\left(r, \tau-t, \theta_{-t} \omega\right)\left\|A^{v+\frac{1}{2}} u_{N}\right\| \\
& \leq K_{8}^{2}\left(r, \tau-t, \theta_{-t} \omega\right)+\frac{1}{2}\left\|A^{v+\frac{1}{2}} u_{N}\right\|^{2}
\end{aligned}
$$


and

$$
\begin{aligned}
& \int_{U}\left|\left[f_{1}(u, x)-f_{1}\left(u_{L}, x\right)\right] A^{2 v} u_{N}\right| d x \\
& \leq c_{32} \int_{U}\left(1+\left|u_{N}\right|^{2}+\left|u_{L}\right|^{2}\right)\left|u_{N}\right|\left|A^{2 v} u_{N}\right| d x \\
& \leq c_{33}\left(\int_{U}\left(1+\left|u_{N}\right|^{2}+\left|u_{L}\right|^{2}\right)^{3}\right)^{\frac{1}{3}}\left(\int_{U}\left|u_{N}\right|^{\frac{6}{3-4 v}}\right)^{\frac{3-4 v}{6}} \\
& \quad \times\left(\int_{U}\left|A^{2 v} u_{N}\right|^{\frac{6}{1+4 v}}\right)^{\frac{1+4 v}{6}} \\
& \leq K_{9}^{2}\left(r, \tau-t, \theta_{-t} \omega\right)+\frac{1}{8}\left\|A^{v+\frac{1}{2}} u_{N}\right\|^{2} .
\end{aligned}
$$

It follows from above that

$$
y_{1}(r)=\left\|A^{v} \varphi_{N}\right\|^{2}+2 \int_{U}\left[f(u, x)-f_{1}\left(u_{L}, x\right)\right] A^{2 v} u_{N} d x,
$$

where

$$
\begin{aligned}
\int_{u}\left[f(u, x)-f_{1}\left(u_{L}, x\right)\right] A^{2 v} u_{N} d x \\
\leq K_{8}^{2}\left(r, \tau-t, \theta_{-t} \omega\right)+\frac{1}{8}\left\|A^{v+\frac{1}{2}} u_{N}\right\|^{2} \\
\quad+K_{9}^{2}\left(r, \tau-t, \theta_{-t} \omega\right)+\frac{1}{8}\left\|A^{v+\frac{1}{2}} u_{N}\right\|^{2} \\
\leq K_{10}^{2}\left(r, \tau-t, \theta_{-t} \omega\right)+\frac{1}{4}\left\|A^{v+\frac{1}{2}} u_{N}\right\|^{2} .
\end{aligned}
$$

So we obtain

$$
\begin{aligned}
\left\|A^{v} \varphi_{N}\right\| & =\left\|A^{v+\frac{1}{2}} u_{N}\right\|^{2}+\left\|A^{v} u_{N, t}\right\|^{2} \\
& \leq y_{1}\left(r, \tau-t, \theta_{-t} \omega, \varphi_{\tau-t}\left(\theta_{-t} \omega\right)\right)+K_{10}^{2}\left(r, \tau-t, \theta_{-t} \omega\right) \\
& \leq \alpha^{2} M_{8}(\omega)+M_{9}+K_{10}^{2}\left(r, \tau-t, \theta_{-t} \omega\right) \leq M_{N}^{2}(\omega)
\end{aligned}
$$

The proof is completed.

Lemma 5 For any $\tau \in R, \omega \in \Omega$, and $t>0$, assume that $B_{v}(\tau, \omega) \subseteq B_{1}(\tau, \omega) \subseteq B_{0}(\omega)$ and $B_{v}(\tau, \omega) \in D_{E^{v}}$, where $v$ is as in Lemma 4 , then if assumptions $\left(H_{1}\right)-\left(H_{2}\right)$ hold, then there exist a random variable $t_{v}(\omega)>0$ and a tempered random variable $M_{v}(\omega)>0$ such that, for any $t \geq t_{v}(\omega), \varphi_{\tau-t}\left(\theta_{-t} \omega\right) \subset B_{v}\left(\tau-t, \theta_{-t} \omega\right) \subseteq B_{0}\left(\theta_{-t} \omega\right) \cap D\left(E^{v}\right)$, the solution $\varphi$ of Eqs. (12)(13) satisfies

$$
\begin{aligned}
& \left\|\varphi\left(\tau, \tau-t, \theta_{-\tau} \omega, \varphi_{\tau-t}\left(\theta_{-\tau} \omega\right)\right)\right\|_{E^{\nu}}^{2} \\
& \quad=\left\|A^{v} \varphi\left(\tau, \tau-t, \theta_{-\tau} \omega, \varphi_{\tau-t}\left(\theta_{\tau-t} \omega\right)\right)\right\|_{E}^{2} \leq M_{\nu}^{2}(\omega) .
\end{aligned}
$$


Proof Taking the inner product of Eqs. (12)-(13) in $E$ with $A^{2 v} \varphi=\left(A^{2 v} u, A^{2 v} v\right)^{T}$, then for any $r \geq \tau-t$, we have

$$
\begin{aligned}
\frac{1}{2} \frac{d}{d t} & \left(\left\|A^{2 v} \varphi\right\|_{E}^{2}+2 \int_{U} f(u, x) A^{2 v} u d x\right)+\left(Q \varphi, A^{2 v} \varphi\right)_{E} \\
& +\varepsilon\left(f(u, x), A^{2 v} \varphi\right)+\left(f(u, x), a A^{2 v} h(x) z\left(\theta_{r-\tau} \omega\right)\right) \\
= & \left(a h(x) z\left(\theta_{r-\tau} \omega\right), A^{2 v} u\right)+(g(x, r) \\
& \left.+(\varepsilon-\alpha+1) a h(x) z\left(\theta_{r-\tau} \omega\right), A^{2 v} v\right) .
\end{aligned}
$$

The same to (75), the following inequality holds:

$$
\begin{aligned}
\left|\int_{U} f(u, x) A^{2 v} u d x\right| & \leq c_{3} \int_{U}\left|\left(1+u^{4}\right)\right|\left|A^{2 v} u\right| d x \\
& \leq c_{3}\left(\int_{U}\left(1+u^{4}\right)^{\frac{6}{5-4 v}} d x\right)^{\frac{5-4 v}{6}}\left(\int_{U}\left|A^{2 v} u\right|^{\frac{6}{1+4 v}}\right)^{\frac{1+4 v}{6}} \\
& \leq c_{3} M_{6}\left(\theta_{r-\tau} \omega\right)+\frac{\mu}{4}\left\|A^{v+\frac{1}{2}} u\right\|^{2}
\end{aligned}
$$

Similar to (72), by (80) and (81), we get

$$
\frac{d}{d t} y_{2}+\sigma_{1} y_{2} \leq \beta_{3}\left(\theta_{r-\tau} \omega\right)
$$

where

$$
\begin{aligned}
& y_{2}=\left\|A^{v} \varphi(r)\right\|_{E}^{2}+2 \int_{U} f(u, x) A^{2 v} d x \geq \frac{1}{2}\left\|A^{v} \varphi(r)\right\|_{E}^{2}-c_{3} M_{6}\left(\theta_{r-\tau} \omega\right), \\
& \beta_{3}=K_{11}\left(r, \tau-t, \theta_{t} \omega\right)+c_{22} a^{2} z^{2}\left(\theta_{r-\tau} \omega\right) \\
& y_{2}(r) \leq y_{2}(\tau-t) e^{-\sigma_{1}(r+t-\tau)}+\int_{\tau-t}^{r} \beta_{3}\left(\theta_{\xi-\tau} \omega\right) e^{-\sigma_{1}(r-\xi)} d \xi
\end{aligned}
$$

By applying Gronwall's inequality to (82) on $[\tau-t, r]$, one has

$$
\begin{aligned}
& \left\|A^{v} \varphi\left(\tau, \tau-t, \theta_{-\tau} \omega, \varphi_{\tau-t}\left(\theta_{-\tau} \omega\right)\right)\right\| \\
& \leq 2 y_{2}\left(\tau, \tau-t, \theta_{-\tau} \omega, \varphi_{\tau-t}\left(\theta_{-\tau} \omega\right)\right)+c_{3} M_{6}(\omega) \\
& \leq 2 y_{2}(\tau-t) e^{-\sigma_{1} t}+2 \int_{\tau-t}^{r} \beta_{3}\left(\theta_{\xi-t} \omega\right) e^{-\sigma_{1}(\tau-\xi)} d \xi+4 c_{3} M_{6}(\omega) \\
& \leq 2 y_{2}(\tau-t) e^{-\sigma_{1} t}+2\left[\int_{-\tau}^{0}\left(K_{11}\left(r, \tau-t, \theta_{t} \omega\right)+c_{22} a^{2} z^{2}\left(\theta_{r-\tau} \omega\right)\right] e^{\sigma_{1} \xi} d \xi\right. \\
& \quad+4 c_{3} M_{6}(\omega) .
\end{aligned}
$$

From (81), (83), (85), and $\varphi_{\tau-t}\left(\theta_{-\tau} \omega\right) \in B_{0}\left(\theta_{-t} \omega\right) \cap D(E)$, it is clear that as $t \rightarrow+\infty$,

$$
y_{2}(\tau-t) e^{-\sigma_{1} t} \leq\left(\frac{3}{2}\left\|A^{v} \varphi_{\tau-t}\right\|\right)_{E}^{2}+2 c_{3} M_{6}\left(\theta_{-t} \omega\right) e^{-\sigma_{1} t} \rightarrow 0
$$


Taking

$$
M_{v}^{2}=4 c \int_{-\infty}^{0}\left(K_{11}\left(r, \tau-t, \theta_{t} \omega\right)+c_{22} a^{2} z^{2}\left(\theta_{r-\tau} \omega\right)\right] e^{\sigma_{1} \xi} d \xi+8 c_{3} M_{6}(\omega)
$$

then the proof is completed.

\section{Existence of random attractor}

Lemma 6 If assumptions $\left(H_{1}\right)-\left(H_{2}\right)$ hold, then for any $\tau \in R, \omega \in \Omega$, there exist $T_{v}(\omega) \geq 0$, a random bounded ball $\hat{B}_{1}$ of $E$, a positive number $\hat{\rho}$, and a tempered random variable $\hat{Q}(\omega)$ such that, for any $t \geq T_{\nu}(\omega)$ and $\varphi_{\tau-t}\left(\theta_{-\tau} \omega\right) \in B_{1}\left(\tau-t, \theta_{-t} \omega\right)$, the solution $\varphi$ of (12)(13) satisfies

$$
d_{E}\left(\varphi\left(\tau, \tau-t, \theta_{-\tau} \omega, B_{1}\left(\tau-t, \theta_{-t} \omega\right)\right), \hat{B}_{1}(\omega)\right) \leq \hat{Q}\left(\theta_{-t} \omega\right) e^{-\hat{\rho} t}
$$

By Lemma 7.6 in [2], Lemma 3, and Lemma 4, one can prove Lemma 6. Since the proof of Lemma 6 is similar to that of Lemma 3.8 in [29], we omit it here. From Lemmas 5 and 6 , it is easy to see the existence of a random attractor for the cocycle $\Phi$.

Theorem 2 If assumptions $\left(H_{1}\right)$ and $\left(H_{2}\right)$ hold, then the cocycle $\Phi$ associated with (12)(13) possesses a $D(E)$-pullback random attractor $A \in D(E)$ such that, for any $\tau \in R, \omega \in \Omega$, $A(\tau, \omega) \subseteq \hat{B_{1}}(\omega) \cap B_{0}(\omega)$, where $B_{0}(\omega)$ and $\hat{B}_{1}(\omega)$ are as in (45) and Lemma 6 , respectively.

Proof For any $\tau \in R$ and $\omega \in \Omega$, by Lemma 6 and the compactness of embedding $E^{\nu} \rightarrow E$, $\hat{B}_{1}(\omega)$ is a compact measurable $D(E)$-pullback attracting ball in $E$. By Proposition 2.1, the cocycle $\Phi$ has a $D(E)$-pullback random attractor $\mathcal{A} \in D(E)$ such that, for any $\tau \in R, \omega \in \Omega$, $\mathcal{A}(\tau, \omega) \subseteq \hat{B}_{1}(\omega) \cap B_{0}(\omega)$. The proof is completed.

\section{Acknowledgements}

The authors would like to thank the anonymous referees for helpful comments and suggestions that improved the original paper.

\section{Funding}

The authors are supported by the National Natural Science Foundation of China (11872264).

\section{Availability of data and materials}

No data, models, or code are generated or used during the study.

Competing interests

The authors declare that they have no competing interests.

Authors' contributions

HY completed the proofs of the main theorems, the rest of this paper was accomplished by JZ. All authors read and approved the final manuscript.

\section{Author details}

${ }^{1}$ College of Mechanical and Vehicle Engineering, Taiyuan University of Technology, Taiyuan, P.R. China. ${ }^{2}$ College of Mathematics, Taiyuan University of Technology, Taiyuan, P.R. China.

\section{Publisher's Note}

Springer Nature remains neutral with regard to jurisdictional claims in published maps and institutional affiliations. 


\section{References}

1. Fall, D., You, Y.: Global attractors for the damped nonlinear wave equation in unbounded domains (2017). arXiv:1801.00104

2. Ding, P., Yang, Z., Li, Y.: Global attractor of the Kirchhoff wave models with strong nonlinear damping. Appl. Math. Lett. 76, 40-45 (2018)

3. Wang, M., Huang, J.: Finite dimensionality of the global attractor for a fractional Schrödinger equation on R. Appl. Math. Lett. 98, 432-437 (2019)

4. Zelik, S.: Asymptotic regularity of solutions of a non-autonomous damped wave equation with a critical growth exponent. Commun. Pure Appl. Anal. 3, 921-934 (2004)

5. Caraballo, T., Lukaszewicz, G., Real, J.: Pullback attractors for asymptotically compact non-autonomous dynamical systems. Nonlinear Anal., Theory Methods Appl. 64(3), 484-498 (2006)

6. Yang, L., Zhong, C.K.: Uniform attractor for non-autonomous plate equations with a localized damping and a critical nonlinearity. Nonlinear Anal. 338, 1243-1254 (2008)

7. Wang, M., Chang, W., Li, X.: Uniform attractor for a non-autonomous parabolic equation with nonlocal diffusion and delay. J. Adv. Appl. Math. 3(2), 50-61 (2018)

8. Hoang, L.T., Olson, E.J., Robinson, J.C.: Continuity of pullback and uniform attractors. J. Differ. Equ. 264(6), 4067-4093 (2018)

9. Crauel, H., Debussche, A.: Random attractors. J. Dyn. Differ. Equ. 9, 307-341 (1997)

10. LV, Y., Wang, W.: Limiting dynamics for stochastic wave equations. J. Differ. Equ. 244, 1-23 (2008)

11. Shen, Z., Zhou, S., Shen, W.: One-dimensional random attractor and rotation number of the stochastic damped sine-Gordon equation. J. Differ. Equ. 248, 1432-1457 (2010)

12. Wang, Z.J., Zhou, S.F., Gu, A.H.: Random attractor for a stochastic damped wave equation with multiplicative noise on unbounded domains. Nonlinear Anal., Real World Appl. 12, 3468-3482 (2011)

13. Yang, M.H., Duan, J., Kloeden, P.: Asymptotic behavior of solutions for random wave equation with nonlinear damping and white noise. Nonlinear Anal., Real World Appl. 12, 464-478 (2011)

14. Wang, B.: Asymptotic behavior of stochastic wave equations with critical exponents on $R^{3}$. Trans. Am. Math. Soc. 363, 3639-3663 (2001)

15. Wang, B.: Upper semicontinuity of random attractors for non-compact random dynamical systems. Electron. J. Differ. Equ. 2009, Article ID 139 (2009)

16. Wang, B.: Sufficient and necessary criteria for existence of pullback attractors for non-compact random dynamical systems. J. Differ. Equ. 253, 1544-1583 (2012)

17. Wang, B.: Random attractors for non-autonomous stochastic wave equations with multiplicative noise. Discrete Contin. Dyn. Syst. 34, 269-298 (2014)

18. Li, H.Y., You, Y.C., Tu, J.Y.: Random attractors and averaging for non-autonomous stochastic wave equations with nonlinear damping. J. Differ. Equ. 258, 148-190 (2015)

19. Wang, Z.J., Zhang, L.P.: Finite fractal dimension of random attractor for stochastic non-autonomous strongly damped wave equation. Comput. Math. Appl. 75, 3343-3357 (2018)

20. Wang, Z., Zhou, S.: Random attractors for non-autonomous stochastic strongly damped wave equation on unbounded domains. J. Appl. Anal. Comput. 5, 363-387 (2015)

21. Wang, Z.J., Liu, Y.N.: Existence and upper semicontinuity of random attractors for non-autonomous stochastic strongly damped sine-Gordon equation on unbounded domains. Comput. Math. Appl. 73, 1445-1460 (2017)

22. Lu, H., Qi, J., Wang, B., et al.: Random attractors for non-autonomous fractional stochastic parabolic equations on unbounded domains. Discrete Contin. Dyn. Syst., Ser. A 39, 683-706 (2019)

23. Zhou, S., Zhao, M.: Random attractors for damped non-autonomous wave equations with memory and white noise. Nonlinear Anal. 120, 202-226 (2015)

24. Arnold, L.: Random Dynamical Systems. Springer, New York (1998)

25. Chueshov, I.: Monotone Random Systems Theory and Applications. Springer, New York (2002)

26. Fan, X.M.: Random attractor for a damped sine-Gordon equation with white noise. Pac. J. Math. 216, 63-76 (2004)

27. Fan, X.M., Wang, Y.G.: Fractal dimensional of attractors for a stochastic wave equation with nonlinear damping and white noise. Stoch. Anal. Appl. 25, 381-396 (2007)

28. Pazy, A.: Semigroups of Linear Operators and Applications to Partial Differential Equations. Springer, New York (1983)

29. Zhou, S., Zhao, M.: Fractal dimension of random attractor for non-autonomous random dynamical systems and random attractor for stochastic damped wave equation. Nonlinear Anal. 133, 292-318 (2016)

\section{Submit your manuscript to a SpringerOpen ${ }^{\circ}$ journal and benefit from:}

- Convenient online submission

- Rigorous peer review

- Open access: articles freely available online

- High visibility within the field

- Retaining the copyright to your article

Submit your next manuscript at $\boldsymbol{s p r i n g e r o p e n . c o m ~}$ 